\title{
Blind Mobile Positioning in Urban Environment Based on Ray-Tracing Analysis
}

\author{
Shohei Kikuchi, ${ }^{1}$ Akira Sano, ${ }^{1}$ and Hiroyuki Tsuji ${ }^{2}$ \\ ${ }^{1}$ School of Integrated Design Engineering, Faculty of Science and Technology, Keio University, 3-14-1 Hiyoshi Kohoku-ku, \\ Yokohama, Kanagawa 223-8522, Japan \\ ${ }^{2}$ Wireless Communications Department, National Institute of Information and Communications Technology (NICT), \\ 3-4 Hikarino-Oka, Yokosuka, Kanagawa 239-0847, Japan
}

Received 1 June 2005; Revised 27 October 2005; Accepted 13 January 2006

\begin{abstract}
A novel scheme is described for determining the position of an unknown mobile terminal without any prior information of transmitted signals, keeping in mind, for example, radiowave surveillance. The proposed positioning algorithm is performed by using a single base station with an array of sensors in multipath environments. It works by combining the spatial characteristics estimated from data measurement and ray-tracing (RT) analysis with highly accurate, three-dimensional terrain data. It uses two spatial parameters in particular that characterize propagation environments in which there are spatially spreading signals due to local scattering: the angle of arrival and the degree of scattering related to the angular spread of the received signals. The use of RT analysis enables site-specific positioning using only a single base station. Furthermore, our approach is a so-called blind estimator, that is, it requires no prior information about the mobile terminal such as the signal waveform. Testing of the scheme in a city of high density showed that it could achieve $30 \mathrm{~m}$ position-determination accuracy more than $70 \%$ of the time even under non-lineof-sight conditions.
\end{abstract}

Copyright @ 2006 Hindawi Publishing Corporation. All rights reserved.

\section{INTRODUCTION}

Interest in determining the position of wireless terminals has been growing rapidly for a number of wireless applications, such as location-based services, navigation, and security. In the United States, for example, the Federal Communications Commission (FCC) requires wireless carriers implementing enhanced 911 (E-911) service to provide estimates of a caller's location within a given accuracy, for instance, wireless E-911 callers have to be located within $50 \mathrm{~m}$ of their actual location at least $67 \%$ of the time [1-3]. In Japan, there is a need to determine the locations of illegal wireless terminals on vehicles that are interfering with wireless communication systems [4]. Position determination is also needed for radiowave surveillance. The most widely used positiondetermination scheme is the global positioning system (GPS) [5]. Although it can be used to determine the locations of things highly accurately, existing handsets have to be modified to function as a GPS receiver, and it does not work unless the mobile terminal has a line-of-sight (LOS) path to the satellites [2]. Thus, it is not applicable to the detection of a nonsubscriber such as the radiowave surveillance.

In a few decades, the use of array antennas is receiving much attention through the efficient use of information carried in the spatial dimension $[1,6]$. More and more mobile positioning schemes using array antenna employed at a high base station have been investigated as the number of cellular handset subscribers increases. Until now, a number of conventional position-determination methods have been based on trilateration, which combines the received signal strength (RSS), time-of-arrival (TOA), time-delay-of-arrival (TDOA), and/or angle-of-arrival (AOA) of signals received at three receivers, for example, see [7-10]. This approach also depends on there being an LOS path between each receiver and transmitter, which is difficult to observe in urban environments since a non-LOS (NLOS) condition significantly degrades positioning accuracy. Although some NLOS mitigation strategies can partly improve accuracy by exploiting $a$ priori knowledge or using a sensor network to a certain extent $[11,12]$, the propagation characteristics greatly depend on the measurement area and the location of the transmitters and receivers.

On the other hand, database correlation methods, socalled fingerprint methods, have been showing better detection capability rather than the trilateration in the last couple of years, see [13-16] and the references therein. The received signal fingerprints, such as RSS, TDOA, and angular profile, are stored as a database by actual measurement in a testing 
area, and the estimated location is obtained by minimizing the Euclidean distance between a sample signal vector and the location fingerprints in the database. This site-specific technique is especially popular in indoor location systems such as existing wireless local area network (WLAN) infrastructure [14]. The straightforward extension to outdoor positioning in general cellular systems is unrealistic considering an immense amount of time and effort to make a database [16]. Furthermore, the dynamic nature of the outdoor radio environments makes fingerprint methods infeasible. Instead of the database made from measurement data, a model-based approach is promising for outdoor positioning, for example, the use of ray-tracing (RT) analysis that the radiowave propagation in a testing area is virtually simulated by modeling three-dimensional (3D) terrain data and propagation laws. Ahonen and Eskelinen virtually predicted the site-specific fingerprints of a testing area by using the RT analysis, and compared RSSs of received signals with those of the RT analysis results obtained at 7 base stations (a serving cell and 6 strongest neighbors) [13]. Basically, however, the use of the RSSs is not adequate to the applications such as surveillance of illegal wireless terminals and emergency calls from nonsubscribers, since the RSS estimation needs prior information of transmitted signals [10]. Furthermore, using fewer base stations is important from the economic standpoint. Although a positioning algorithm with a single base station employing sensors of array was proposed [17], it utilized the temporal information of impinging signals that also require prior knowledge of transmitted signals $[9,18]$.

This work presents a novel positioning method for use in multipath environments, which has three important features as follows.

(i) It uses a "blind algorithm," that is, it needs no prior information about the transmitted signal, such as its signal waveform.

(ii) It is site-specific in that it takes the propagation environment into consideration by using RT analysis, and pinpoints the location of a terminal using only a single base station.

(iii) It exploits the characteristics of radiowave propagation in urban environments considering a local scattering model.

The algorithm consists of two steps. First, the parameters characterizing the locations in the testing area (defined later) are experimentally estimated from received signals. Second, the RT simulations are virtually conducted for calculating the parameters corresponding to those in the measurement data analysis, and the estimated location is determined by matching with the experimentally estimated parameters. The preliminary calculation of the RT analysis reduces the computational load; however, note that the use of the RT analysis makes a difference from the conventional fingerprint methods in that the fingerprint does not always have to be stored in advance. Furthermore, one of the notable features of the proposed algorithm is to give a blind algorithm in order to meet more variable requirements of positioning issues such as surveillance of illegal wireless terminals as mentioned

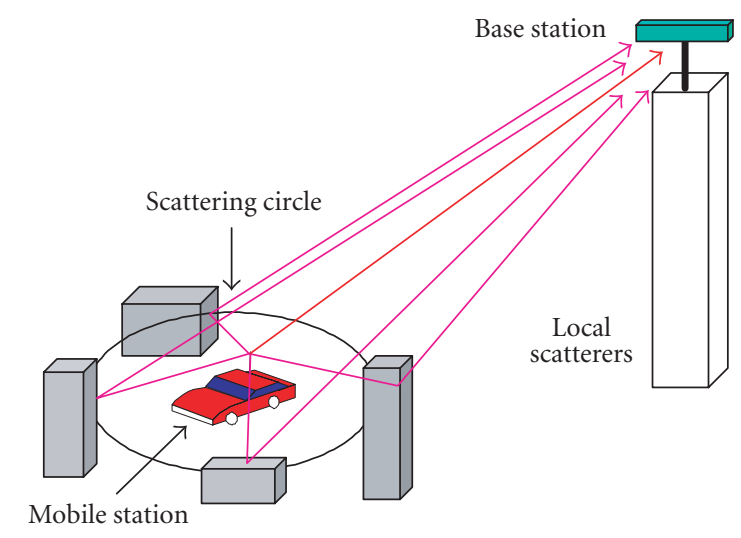

FIGURE 1: Conceptual diagram of local scattering.

above. The estimation of only spatial parameters realizes the blind algorithm, while temporal parameter estimation needs prior information of signal waveform [18]. Those using code-division multiple access (CDMA), like those described by Caffery and Stüber [7], are also considerable for future communication systems, and the proposed positioning algorithm can be applied to the narrowband CDMA systems, for example, IS-95 [19], if code information for dispreading is known in advance. Another feature is to model the received signal based on a local scattering model that assumes scattering only in the vicinity of a mobile or some reflectors, for example, see $[20,21]$. This signal model is suitable for the propagation environments of urban areas with a high base station and a low mobile terminal. Then in addition to AOAs of the received signals, this work introduces a new spatial parameter indicating the degree of scattering (DOS) related to the angular spread under the assumption of the local scattering model, like in Figures 1 and 2. The two parameters of AOA and DOS are used for pinpointing the location without any information of transmitted signal waveform. The DOS is related to a parameter derived from the first-order approximation of received signal model [20], and the theoretical performance of the DOS will be also derived in this paper. The matching of these two parameters dramatically mitigates the computational burden, compared to the case that angular profile between $-\pi / 2<\theta<\pi / 2$ itself is used for matching [22]. Furthermore, RT analysis [23] using highly accurate, $3 \mathrm{D}$ terrain data realizes site-specific positioning using only a single base station. Note that the RT analyzer follows the fundamental property of radiowave propagation, for example, geometrical optics (GO) and uniform theory of diffraction (UTD) [24].

In this paper, the effectiveness of the proposed positioning method is evaluated through experimental data analysis measured at Yokosuka City in Japan, and the results show that the combination of measurement data and RT analysis and exploitation of the AOA and DOS prominently improves the positioning accuracy although the test range is limited to approximately $500 \mathrm{~m} \times 500 \mathrm{~m}$. This paper is organized as follows. Section 2 outlines the basic concept of the proposed position-determination scheme. The method for estimating 


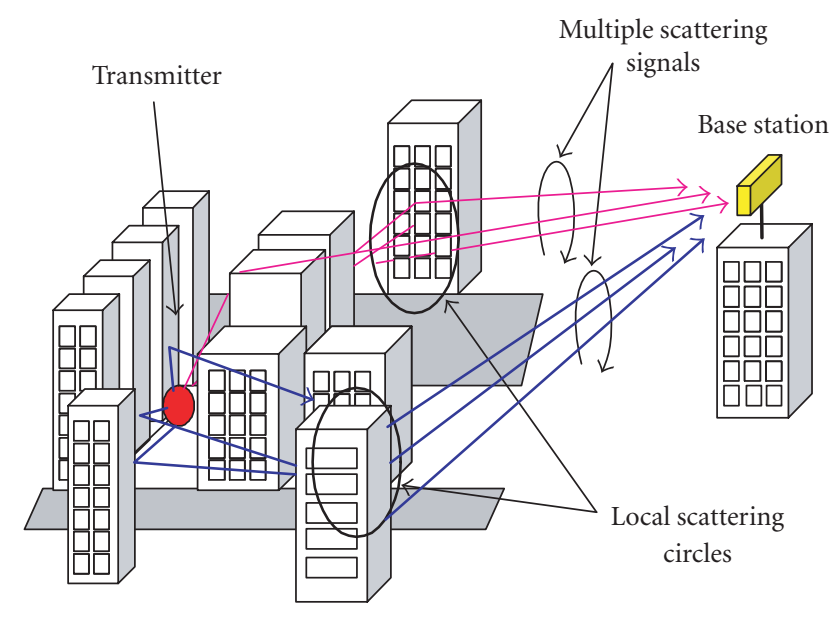

Figure 2: Local scattering on reflectors.

the AOA and DOS in the experimental data analysis and the theoretical behavior of the DOS are described in Section 3. Section 4 mentions the fundamental property of the RT analysis and how to exploit the parameters corresponding to the AOA and DOS from the RT analysis result. The parameter estimation results obtained through experimental data analysis and the positioning accuracy of the proposed algorithm are discussed in Section 5. We conclude in Section 6 with a brief summary.

\section{CONCEPT OF PROPOSED POSITION-DETERMINATION SCHEME}

\subsection{Local scattering model and parameters characterizing terminal location}

Suppose that a transmitter in a general cellular system is located in low positions outdoors and its scattered signals, which deteriorate as a result of multipath propagation, are measured at a receiver mounted on top of a building. If the receiver is much higher than the transmitters, a local scattering model, like the one described by Asztély and Ottersten [20], that considers reflections and scattering in the vicinity of each transmitter is an appropriate model of the received signals. In such a model, spatially spread signals are observed at the receiver, as illustrated in Figure 1. However, in practical situations, especially under NLOS conditions between the transmitter and receiver, which is the case dealt with throughout this paper, the spread signals are usually measured after propagating along several routes, as illustrated in Figure 2. As a result, the received signal is expressed as the summation of several local scatterers on some reflections. For example, if there is an LOS between a transmitter and a receiver, the transmitter lies along the AOAs of the direct paths to multiple base stations. If there is no LOS, the locations of terminals cannot always be identified by using the AOA estimates, making the position-determination more difficult.

Our proposed positioning method, using a single array of sensors, uses two particular spatial parameters, the AOA and
DOS, to determine the location of a terminal. These parameters represent the path characteristics, which depend on the propagation environment between the transmitter and receiver. The signals can be discriminated using the DOSs, even if their AOAs are the same. Estimation of these two parameters and the relationship between the angular spread and the bit error rate (BER) are described elsewhere $[25,26]$.

\subsection{Positioning method using ray-tracing analysis}

The AOA and DOS estimated from the received signals are not sufficient for determining the location of a mobile terminal with a single base station, since the location of the mobile is not always determined by such trilateration because of an NLOS condition and/or multipath propagation. We also have to use RT analysis. Using an RT simulator, we can virtually analyze the radiowave propagation using the given terrain data and some propagation parameters such as coefficients of reflection and diffraction. Since the rendering of geographical information has been attracting much attention, this technology should become widely used in a variety of applications in the near future. This work thus uses the RT analysis with highly accurate 3D terrain data around the testing area to estimate the location of a terminal, by comparing with the results of the two spatial parameters from both experimental and RT analyses. In the RT analysis, these parameters can be calculated from all the rays between a transmitter and receiver as shown in Figure 3. In addition, the estimated AOAs and DOSs are virtually measured at all outdoor locations (e.g., every $10 \mathrm{~m}$ ). The calculated AOAs and DOSs in the RT analysis are used for estimating the location of the terminal. Let $\hat{\theta}_{k}$ and $\hat{\eta}_{k}, k=1, \ldots, K$, denote, respectively, the estimated AOA and DOS of the $k$ th scatterer obtained in the experimental analysis. Similarly, let $\theta_{k}^{(\mathrm{RT})}(X, Y)$ and $\eta_{k}^{(\mathrm{RT})}(X, Y), k=1, \ldots, K$, be, respectively, the estimates in the RT analysis, where $(X, Y)$ indicates the Cartesian coordinate of the pseudotransmitters inside the testing area $\mathscr{D}$. Note that $K$ is the number of scatterers in Figure 2, not that of the total rays. We estimate the location of a terminal using a cost function:

$$
\begin{aligned}
F(X, Y)=[ & \sum_{k=1}^{K}(1-\nu)\left(\hat{\theta}_{k}-\theta_{k}^{(\mathrm{RT})}(X, Y)\right)^{2} \\
& \left.+\nu\left(\hat{\eta}_{k}-\eta_{k}^{(\mathrm{RT})}(X, Y)\right)^{2}\right]^{1 / 2},
\end{aligned}
$$

where $\theta_{k}$ is the radian measure, and $0 \leq v \leq 1$ is a weighting factor that indicates the ratio between the correlation of the AOAs and DOSs. The $(X, Y)$ minimizing this cost function is taken as the estimated position. That is,

$$
(\hat{X}, \hat{Y})=\arg \min _{X, Y \subseteq D} F(X, Y),
$$

where $(\hat{X}, \hat{Y})$ is the estimated position. The diagram of this algorithm is illustrated in Figure 4. Combining the results for multiple signals from different directions enables to use the multipath propagation, conventionally regarded as a 


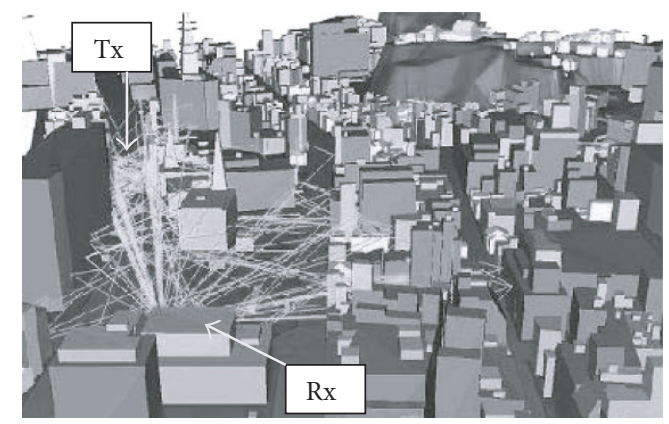

Figure 3: 3D terrain data around testing area and RT analysis. A number of rays from a transmitter $(\mathrm{Tx})$ reach a receiver $(\mathrm{Rx})$ via different reflections and diffractions.

problem to be avoided, to pinpoint the locations of mobile terminals using only a single receiver even under NLOS conditions.

Remark 1. This work deals with the position determination of one mobile terminal using a single base station. If the number of users is more than one, then the total number of scatterers is $K_{T}=\sum_{i=1}^{I} K_{i}$, where $I$ denotes the number of transmitted sources, and $K_{i}$ is the number of scatterers generated from the $i$ th source. In order to determine the position of the mobiles, we need the identification of $\left\{K_{i}\right\}_{i=1}^{I}$ and the association, that is, which transmitted source the $k$ th scatterer belongs to. This problem is called "source association." As one idea to solve the problem, Yan and Fan proposed an algorithm for categorizing the distinct $K_{T}$ AOAs into $I$ groups in the case that the $i$ th group includes $K_{i}$ coherent signals [27]. Note that the total number of scatterers $K_{T}$ has to meet the condition $M>K_{T}$, where $M$ is the number of sensors of array. Suppose $I=1$ and $K_{T}=K_{1} \triangleq K$ throughout this paper.

\section{DATA MODEL AND PARAMETER ESTIMATION}

This section describes the received signal model for multipath environments, like the one illustrated in Figure 2, based on the local scattering model. We also mention the estimation of the AOA and DOS, and statistically derive the physical properties of the DOS.

\subsection{Signal model considering local scattering}

The received signal model is expressed as the summation of multiple local scatterers $[25,26,28]$. We assume that the transmitter is stationary during observation and that the time dispersion introduced by the multipath propagation is small compared to the reciprocal of the bandwidth of the transmitted signals. An $M$-element uniform linear array (ULA) is used as the base station; it is mounted on top of a high building. A flat Rayleigh fading narrowband channel is considered. The received signal consists of $K$ scatterers; the number depends on the physical propagation phenomena, such as reflection and diffraction:

$$
\begin{aligned}
\mathbf{x}(t) & =\sum_{k=1}^{K} \sum_{l=0}^{L_{k}} \beta_{k l} \mathbf{a}\left(\theta_{k}+\tilde{\theta}_{k l}\right) s\left(t-\tau_{k l}\right)+\mathbf{n}(t) \\
& \approx \sum_{k=1}^{K} \sum_{l=0}^{L_{k}} \alpha_{k l} \mathbf{a}\left(\theta_{k}+\tilde{\theta}_{k l}\right) s_{k}(t)+\mathbf{n}(t),
\end{aligned}
$$

where $L_{k}$ and $\beta_{k l}$ are the total number of rays associated with the $k$ th scatterer and complex amplitude of the $l$ th ray in the $k$ th scatterer, respectively. $s_{k}(t)$ is the signal of the $k$ th scatterer, and $\mathbf{n}(t)$ is an additive white Gaussian noise (AWGN) vector. We assume that the array response vector is perfectly known from calibration. The $m$ th factor of $\mathbf{a}\left(\theta_{k}\right)$ is expressed as $a_{m}\left(\theta_{k}\right)=\exp \left\{j 2 \pi d \sin \theta_{k} / \lambda\right\}$ for ULAs. The quantities $\theta_{k}$ and $\theta_{k}+\tilde{\theta}_{k l}$ represent the nominal AOA of the $k$ th scatterer and the arrival angle of the $l$ th ray in the $k$ th scatterer, respectively. This means that $\left|\beta_{k 0}\right|$ is sufficiently large compared to $\left|\beta_{k l}\right|$ under the condition that the $k$ th scatterer includes a direct path, while $\left|\beta_{k 0}\right|$ is at almost the same level as $\left|\beta_{k l}\right|$ if the scatterer results from reflections. Note that this model covers both LOS and NLOS conditions. Assuming narrowband signals, the time delay of the scattered signals is included in the phase shift [20]. Thus, given the definitions $s_{k}(t) \triangleq s\left(t-\tau_{k 0}\right)$ and $\Delta \tau_{k l} \triangleq \tau_{k l}-\tau_{k 0}$, we obtain (4) from (3) using an approximation:

$$
\begin{gathered}
s\left(t-\tau_{k l}\right) \approx s_{k}(t) \exp \left(-j 2 \pi f_{c} \Delta \tau_{k l}\right), \\
\alpha_{k l}=\beta_{k l} \exp \left(-j 2 \pi f_{c} \Delta \tau_{k l}\right), \quad k=1, \ldots, K .
\end{gathered}
$$

\subsection{Scattering parameter}

\subsubsection{Definition}

It is impossible to identify all the unknown parameters in (4) since the number of scattered signals, $L_{k}$, is too large and uncountable. Therefore, a number of statistical approaches to deal with the scattering model have been so far proposed. For instance, the standard deviation of the distributed rays is estimated by the weighted subspace fitting [21], which requires heavy computational load. On the other hand, assuming that the rays are independent and identically distributed with phases uniformly distributed over $[0,2 \pi]$, and that the number of rays is sufficiently large, the central limit theorem may be used to approximate the elements of the spatial signature as complex Gaussian random variables. Thus, (4) can be approximated using a first-order Taylor expansion under the assumption that the angular spread is small, that is, $\left|\tilde{\theta}_{k l}\right| \rightarrow 0$ $[20,21]$ :

$$
\begin{aligned}
\mathbf{x}(t) & \approx \sum_{k=1}^{K} \sum_{l=0}^{L_{k}} \alpha_{k l}\left[\mathbf{a}\left(\theta_{k}\right)+\tilde{\theta}_{k l} \mathbf{d}\left(\theta_{k}\right)\right] s_{k}(t)+\mathbf{n}(t) \\
& =\sum_{k=1}^{K}\left[\gamma_{k} \mathbf{a}\left(\theta_{k}\right)+\phi_{k} \mathbf{d}\left(\theta_{k}\right)\right] s_{k}(t)+\mathbf{n}(t) \\
& =\sum_{k=1}^{K}\left[\mathbf{a}\left(\theta_{k}\right)+\rho_{k} \mathbf{d}\left(\theta_{k}\right)\right] \tilde{s}_{k}(t)+\mathbf{n}(t),
\end{aligned}
$$




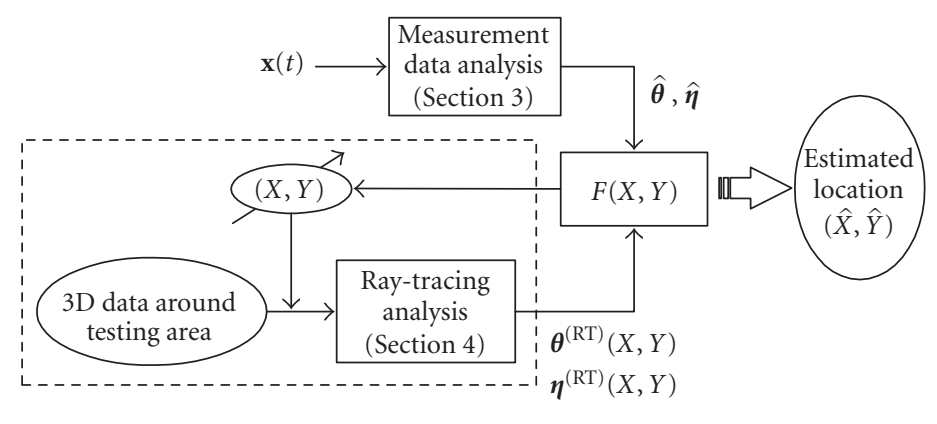

FIGURE 4: Diagram of proposed positioning algorithm.

where $\mathbf{d}(\theta) \triangleq \partial \mathbf{a}(\theta) / \partial \theta$, and

$$
\gamma_{k} \triangleq \sum_{l=0}^{L_{k}} \alpha_{k l}, \quad \phi_{k} \triangleq \sum_{l=0}^{L_{k}} \alpha_{k l} \widetilde{\theta}_{k l}
$$

Including $\gamma_{k}$ in $s_{k}(t)$ as the complex amplitude, we define $\rho_{k} \triangleq \phi_{k} / \gamma_{k}$ and $\tilde{s}_{k}(t) \triangleq \gamma_{k} s_{k}(t)$. Due to the definitions of $\gamma_{k}$ and $\phi_{k}$ of (7), the identification of the number of the rays in a scatterer $L_{k}$ is unnecessary. The model is then consistent with flat Rayleigh fading since the magnitude of each element of the spatial signature has a Rayleigh distribution. There are three unknown parameters in $(6), \theta_{k}, \rho_{k}$, and $\widetilde{s}_{k}(t) ; \rho_{k}$ has been discussed elsewhere $[20,25]$. Actually, however, $\rho_{k}$ temporally fluctuates as a result of multipath fading in practical situations. Thus, we define a new parameter called the "degree of scattering (DOS)" using the expectations of the absolute values of $\phi_{k}$ and $\gamma_{k}$ as

$$
\eta_{k} \triangleq \frac{E\left\{\left|\phi_{k}\right|\right\}}{E\left\{\left|\gamma_{k}\right|\right\}}
$$

where $E\{\cdot\}$ denotes the expectation. This parameter $\eta_{k}$ is theoretically relevant to the angular spread of the $k$ th scatterer, and the detailed behavior of the parameter is discussed in Section 3.2.3. The DOS can be estimated without any prior information such as signal waveform, and the identification of both AOA and DOS is appropriate for fingerprint to determine the location under the assumption of the local scattering model.

\subsubsection{Parameter estimation method}

To estimate the AOAs and DOSs, we assume that the number of scatterers $K$ is correctly estimated in advance. Although eigenvalue-based nonparametric source number detection methods such as the Akaike information criterion (AIC) and minimum description length (MDL) criterion are commonly used [29], they does not work well in the presence of angular spread. Recently, robust source number estimators have been described elsewhere, for example, [30], based on the generalized maximum-likelihood-ratio test principles, that work well even for slightly scattered signals. The $K$ nominal AOAs are estimated from correlated sources by an AOA localizer based on TLS-ESPRIT [31] with a spatial smoothing [32], under the assumption that the angular distribution for a scatterer is symmetrical. The DOSs are obtained using the leastsquares (LSs) method:

$$
\left[\hat{\widetilde{s}}_{k}(t), \hat{\rho}_{k}\right]=\arg \min _{\widetilde{s}_{k}(t), \rho_{k}} J(t),
$$

where $J(t)$ is the cost function used to estimate $\widehat{\widetilde{s}}_{k}(t)$ and $\hat{\rho}_{k}$,

$$
J(t)=\left|\mathbf{x}(t)-\sum_{k=1}^{K}\left[\mathbf{a}\left(\hat{\theta}_{k}\right)+\rho_{k} \mathbf{d}\left(\hat{\theta}_{k}\right)\right] \tilde{s}_{k}(t)\right|^{2} .
$$

The $K$ sets of DOS are recursively calculated using only the $\mathbf{x}(t)$ of the received signals as follows.

Step 1. Obtain $\hat{\theta}_{k}, k=1, \ldots, K$.

Step 2. Initialize $K$-column vector, $\hat{\boldsymbol{\rho}}^{(0)}=[0, \ldots, 0]^{T}$, where $\hat{\boldsymbol{\rho}}^{(i)}$ denotes the ith iteration of $\hat{\boldsymbol{\rho}}=\left[\hat{\rho}_{1}, \ldots, \hat{\rho}_{K}\right]^{T}$.

Step 3. Calculate ML estimate $\widehat{\widetilde{\widetilde{s}}}_{k}(t)$ :

$$
\hat{\widetilde{\mathbf{s}}}(t)=\left(\hat{\mathbf{V}}^{H} \hat{\mathbf{V}}\right)^{-1} \hat{\mathbf{V}}^{H} \mathbf{x}(t),
$$

where

$$
\hat{\mathbf{V}}=\sum_{k=1}^{K}\left[\mathbf{a}\left(\hat{\theta}_{k}\right)+\hat{\rho}_{k} \mathbf{d}\left(\hat{\theta}_{k}\right)\right], \quad \hat{\widetilde{\mathbf{s}}}(t)=\left[\hat{\widetilde{s}}_{1}(t), \ldots, \hat{\widetilde{s}}_{K}(t)\right]^{T} \text {. }
$$

Step 4. Estimate $\rho_{k}$ using an LS approach that minimizes the following cost function:

$$
J_{2}=E\left\{|\widehat{\mathbf{x}}(t)-\mathbf{x}(t)|^{2}\right\}
$$

where

$$
\begin{gathered}
\hat{\mathbf{x}}(t)=\sum_{k=1}^{K}\left[\mathbf{a}\left(\hat{\theta}_{k}\right)+\rho_{k} \mathbf{d}\left(\hat{\theta}_{k}\right)\right] \widehat{\widetilde{s}}_{k}(t)=\mathbf{A} \hat{\widetilde{S}} \mathbf{e}+\mathbf{D} \hat{\widetilde{\mathbf{S}}} \boldsymbol{\rho}, \\
\mathbf{A}=\left[\mathbf{a}\left(\hat{\theta}_{1}\right), \ldots, \mathbf{a}\left(\hat{\theta}_{K}\right)\right], \\
\mathbf{D}=\left[\mathbf{d}\left(\hat{\theta}_{1}\right), \ldots, \mathbf{d}\left(\hat{\theta}_{K}\right)\right], \\
\hat{\widetilde{\mathbf{S}}}=\operatorname{Diag}\left\{\hat{\widetilde{s}}_{1}(t), \ldots, \hat{\widetilde{s}}_{K}(t)\right\}, \\
\boldsymbol{\rho}=\left[\rho_{1}, \ldots, \rho_{K}\right]^{T} \\
\mathbf{e}=[1, \ldots, 1]^{T} .
\end{gathered}
$$


$\operatorname{Diag}\{\cdot\}$ is a diagonal matrix whose diagonal elements are $\{\cdot\}$. Thus, the cost function (13) can be reobtained as

$$
J_{2}=E\left\{|\mathbf{A} \hat{\widetilde{S}} \mathbf{e}+\mathbf{D} \hat{\widetilde{S}} \boldsymbol{\rho}-\mathbf{x}(t)|^{2}\right\}=E\left\{|\mathbf{D} \hat{\widetilde{S}} \boldsymbol{\rho}-\mathbf{z}|^{2}\right\}
$$

where $\mathbf{z}=\mathbf{x}(t)-\mathbf{A} \hat{\tilde{S}} \mathbf{e}$

Step 5. Repeat Steps 4 and 5 until $\hat{\boldsymbol{\rho}}$ converges.

Step 6. Derive $\left|\hat{\gamma}_{k}\right|$ under the condition $E\left\{s_{k}(t) s_{k}^{*}(t)\right\}=1$ :

$$
E\left\{\tilde{s}_{k}(t) \tilde{s}_{k}^{*}(t)\right\}=E\left\{\gamma_{k} s_{k}(t) s_{k}^{*}(t) \gamma_{k}^{*}\right\}=\left|\gamma_{k}\right|^{2} .
$$

Step 7. Calculate $\hat{\phi}_{k}=\left|\hat{\gamma}_{k}\right|\left|\hat{\rho}_{k}\right|$.

Step 8. Repeat the above steps for every time slot (including enough samples). Determine expectations $E\left\{\left|\hat{\gamma}_{k}\right|\right\}$ and $E\left\{\left|\hat{\phi}_{k}\right|\right\}$ by temporal averaging, and obtain $\hat{\eta}_{k}$ from (8).

\subsubsection{Theoretical behavior of scattering parameter}

The theoretical performance of the proposed parameter $\eta_{k}$ is considered to clarify its physical meaning. The resultant formulations are applied to the RT analysis. First, the theoretical behavior of the expectations $E\left\{\left|\gamma_{k}\right|\right\}$ and $E\left\{\left|\phi_{k}\right|\right\}$ are derived for LOS and NLOS conditions, respectively.

From (16), $\left|\gamma_{k}\right|$ means the amplitude envelope of the signal received at the base station, and it varies based on Nakagami-Rice fading, which has a probability density function (pdf) that follows the Ricean distribution. Note that Nakagami-Rice fading includes Rayleigh fading as a special case. Since the phase of $\alpha_{k l}$ changes randomly during observation, the expected values and variances of $\mathfrak{R}\left\{\alpha_{k l}\right\}$ and $\mathfrak{J}\left\{\alpha_{k l}\right\}$ can be expressed, respectively, as

$$
\begin{gathered}
E\left\{\alpha_{\mathrm{Re}}\right\}=E\left\{\alpha_{\mathrm{Im}}\right\}=0, \\
\operatorname{Var}\left\{\alpha_{\mathrm{Re}}\right\}=E\left\{\alpha_{\mathrm{Re}}^{2}\right\}=\frac{\left|\alpha_{k l}\right|^{2}}{2}, \quad \operatorname{Var}\left\{\alpha_{\mathrm{Im}}\right\}=\operatorname{Var}\left\{\alpha_{\mathrm{Re}}\right\},
\end{gathered}
$$

where $[\cdot]_{\text {Re }}$ and $[\cdot]_{\text {Im }}$ denote, respectively, the real and imaginary parts, and $\operatorname{Var}\{\cdot\}$ is the variance. Let $A_{k}^{2} / 2$ and $\mu_{k}^{2}=$ $L_{k} \cdot \operatorname{Var}\left\{\alpha_{\mathrm{Re}}\right\}=L_{k} \cdot \operatorname{Var}\left\{\alpha_{\mathrm{Im}}\right\}$ be, respectively, the power of the main wave and scattered waves. The Ricean factor is defined as the ratio between their powers [24]:

$$
K_{k} \triangleq \frac{A_{k}^{2}}{2 \mu_{k}^{2}} .
$$

Basically, the propagation scenarios can be classified into LOS and NLOS conditions depending on Ricean factor $K_{k}$. We consider the performance of the DOS in both cases. Since $\left|\gamma_{k}\right|$ follows the Ricean distribution, the expectation $E\left\{\left|\gamma_{k}\right|\right\}$ is

$$
E\left\{\left|\gamma_{k}\right|\right\}=\sqrt{\frac{\pi}{2}} \mu_{k} \exp \left(-K_{k}\right) M\left(\frac{3}{2} ; 1 ; K_{k}\right),
$$

where $M(\cdot)$ denotes Kummer's confluent hypergeometric function [33]. The detailed derivation of (19) is given in the appendix. When $K_{k} \gg 1$, the pdf of $\left|\gamma_{k}\right|$ is an approximately Gaussian distribution since the scattered component orthogonal to the main wave can be neglected. The expected value of $\left|\gamma_{k}\right|$ can be approximated as

$$
E\left\{\left|\gamma_{k}\right|\right\} \approx A_{k}
$$

On the other hand, without a high-powered main wave, that is, under NLOS conditions, the level of the scattered waves is almost the same as that of the main wave. Thus, we define $\mu_{k}^{\prime 2}=A_{k}^{2} / 2+\mu_{k}^{2}$ as the total wave power including the main wave. Since the pdf of $\left|\gamma_{k}\right|$ is approximated by a Rayleigh distribution, the expected value of $\left|\gamma_{k}\right|$ can be then expressed as

$$
E\left\{\left|\gamma_{k}\right|\right\} \approx \sqrt{\frac{\pi}{2}} \mu_{k}^{\prime}=\sqrt{\frac{\pi}{2}} \sqrt{\frac{A_{k}^{2}}{2}+\mu_{k}^{2}}=\sqrt{\frac{\pi}{2}} \mu_{k} \sqrt{K_{k}+1} .
$$

Next, the behavior of $\phi_{k}$ is considered. From (7), the real and imaginary parts of $\phi_{k}$ are, respectively,

$$
\phi_{\mathrm{Re}, k}=\sum_{l=1}^{L_{k}} \alpha_{\mathrm{Re}, k, l} \tilde{\theta}_{k l}, \quad \phi_{\operatorname{Im}, k}=\sum_{l=1}^{L_{k}} \alpha_{\operatorname{Im}, k, l} \tilde{\theta}_{k l},
$$

where $\tilde{\theta}_{k 0}=0$ without loss of generality. Under the assumption that $\tilde{\theta}_{k l}$ and $\alpha_{k l}$ have no correlation, the pdfs of both $\phi_{\mathrm{Re}, k}$ and $\phi_{\mathrm{Im}, k}$ can be approximated as Gaussian distributions. The expectations of $\phi_{\mathrm{Re}, k}$ and $\phi_{\mathrm{Im}, k}$ are given, respectively, as

$$
E\left\{\phi_{\mathrm{Re}, k}\right\}=0, \quad E\left\{\phi_{\operatorname{Im}, k}\right\}=0 .
$$

Thus, their variances are, respectively,

$$
\begin{aligned}
& \operatorname{Var}\left\{\phi_{\mathrm{Re}}\right\}=L_{k} E\left\{\tilde{\theta}^{2}\right\} E\left\{\alpha_{\mathrm{Re}}^{2}\right\}=\mu_{k}^{2} \sigma_{\theta_{k}}^{2}, \\
& \operatorname{Var}\left\{\phi_{\mathrm{Im}}\right\}=L_{k} E\left\{\tilde{\theta}^{2}\right\} E\left\{\alpha_{\mathrm{Im}}^{2}\right\}=\mu_{k}^{2} \sigma_{\theta_{k}}^{2},
\end{aligned}
$$

where $\sigma_{\theta_{k}}$ denotes the standard deviation of the angular distribution, the so-called angular spread [21]. Since the distributions of $\phi_{\operatorname{Re}}$ and $\phi_{\operatorname{Im}}$ are Gaussian, the pdf of $|\phi|=$ $\sqrt{\phi_{\mathrm{Re}}^{2}+\phi_{\mathrm{Im}}^{2}}$ follows the Rayleigh distribution. From (24), the expected value of $\left|\phi_{k}\right|$ is

$$
E\left\{\left|\phi_{k}\right|\right\}=\sqrt{\frac{\pi}{2}} \mu_{k} \sigma_{\theta_{k}} .
$$

As shown by (8), the DOS is defined as the ratio between $E\left\{\left|\gamma_{k}\right|\right\}$ and $E\left\{\left|\phi_{k}\right|\right\}$. Under the condition $K_{k} \gg 1$, that is, an LOS condition, we derive the parameter $\eta_{\text {LOS, } k}$ using (20) and (25):

$$
\eta_{\mathrm{LOS}, k}=\frac{E\left\{\left|\phi_{k}\right|\right\}}{E\left\{\left|\gamma_{k}\right|\right\}} \approx \sqrt{\frac{\pi}{2}} \frac{\mu_{k} \sigma_{\theta_{k}}}{A_{k}}=\sqrt{\frac{\pi}{4}} \frac{\sigma_{\theta_{k}}}{\sqrt{K_{k}}},
$$

where $\eta_{\text {LOS, } k}$ is proportional to $\sigma_{\theta_{k}}$ and inversely proportional to $\sqrt{K_{k}}$. Furthermore, when the level of the main wave is almost the same as that of the scattered waves, which occurs mainly under NLOS conditions, $\eta_{\mathrm{NLOS}, k}$ is given from (21) and (25):

$$
\eta_{\mathrm{NLOS}, k}=\frac{E\left\{\left|\phi_{k}\right|\right\}}{E\left\{\left|\gamma_{k}\right|\right\}} \approx \frac{\sigma_{\theta_{k}}}{\sqrt{K_{k}+1}}
$$


where $\eta_{\mathrm{NLOS}, k}$ is proportional to $\sigma_{\theta_{k}}$, and inversely proportional to $\sqrt{K_{k}+1}$. Equations (26) and (27) mean that the DOS $\eta_{k}$ depends on the Ricean factor $K_{k}$ and angular spread $\sigma_{\theta_{k}}$ of each AOA. This means the larger the DOS is, the more widely the impinging $k$ th signal is distributed, and vice versa. Thus, the DOS is an efficient criterion for describing the degree of scattering.

\section{RAY-TRACING ANALYSIS}

Section 2 described the basic procedure of the proposed positioning method. In our scheme, the AOAs and DOSs obtained by practical data analysis are compared with those by RT analysis using the cost function of (1). This section describes how the parameters are calculated in the RT analysis. We use highly accurate, 3D terrain data for the experimental area. The data is collected for approximately 20 layers per material including the conditions of the dielectric properties regarding the materials of reflectors and the $3 \mathrm{D}$ coordinates obtained within a height accuracy of $\pm 25 \mathrm{~cm}$. The RT analysis follows propagation rules such as the GO and UTD [24], and enables us to determine the position of terminals accurately using site-specific information for the measurement area.

In the analysis, the receiver is virtually located in the same place as in the experiment described in the next section, and the waves propagate following the geometric laws of radiowave propagation. We use the ray-launching method [23] for our RT simulator as it is more tractable and computationally reasonable than the other commonly used approach, that is, the imaging method. The ray-launching method radiates a ray at every angle $\Delta \theta$ from a transmitter, and the path is traced through reflection, transmission, and diffraction points, while the imaging method traces a ray reflection and transmission route connecting a transmission point with a reception point by obtaining an imaging point against a reflection surface. Thus, the implementation of the imaging method is unrealistic as the terrain data become huge. As a result of the RT analysis, an angular profile can be obtained like that shown in Figure 5, which indicates the validity of modeling the received signal using the local scattering model. From the profile, a scatterer is defined as a signal cluster including a nominal ray above $30 \mathrm{~dB}$ and rays 10 degrees around when the least signal level that the receiver detects is set at $0 \mathrm{~dB}$. Therefore, Figure 5 can be regarded as a case of $K=2$. The angular spread of each scatterer is calculated using the second-order statistics:

$$
\sigma_{\theta_{k}}^{(\mathrm{RT})}=\sqrt{\frac{1}{L_{k}^{\prime}} \sum_{l^{\prime}=1}^{L_{k}^{\prime}}\left[\left(\theta_{k l^{\prime}}^{(\mathrm{RT})}-\bar{\theta}_{k}^{(\mathrm{RT})}\right)^{2} \cdot \frac{P_{k l^{\prime}}^{(\mathrm{RT})}}{\bar{P}_{k}^{(\mathrm{RT})}}\right]},
$$

where $\bar{\theta}_{k}^{(\mathrm{RT})}$ and $\bar{P}_{k}^{(\mathrm{RT})}$ are, respectively, the nominal AOA and its power, $\theta_{k l^{\prime}}^{(\mathrm{RT})}$ and $P_{k l^{\prime}}^{(\mathrm{RT})}$ are the AOAs and powers of the scattered waves, respectively, and $L_{k}^{\prime}$ is the total number of both nominal and scattered waves. The theoretical behavior of the DOS derived above says that the DOS depends on the standard deviation of the scattered signals and the Ricean

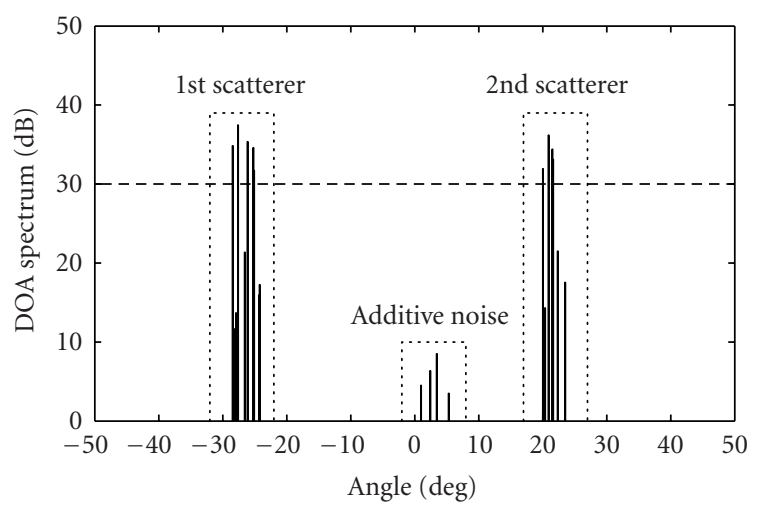

FIGURE 5: Example of angular profile by RT analysis $(K=2)$. It is shown that some rays are launched from the Tx and reflected on the reflector. At the end of the process, a fewer number of rays may be received at the $\mathrm{Rx}$.

factor. Thus, the DOS is also derived from those parameters even in the RT analysis. The Ricean factor is given by $K_{k}^{(\mathrm{RT})}=\bar{P}_{k}^{(\mathrm{RT})} / 2 \sum_{l^{\prime}=1}^{L_{k}^{\prime}} P_{k l^{\prime}}^{(\mathrm{RT})}$ since it is the ratio between the powers of the main and scattered waves. Using (26), (27), and (28), we can obtain the DOS under LOS conditions by

$$
\eta_{\mathrm{LOS}, k}^{(\mathrm{RT})}=\sqrt{\frac{\pi}{4}} \frac{\sigma_{k}^{(\mathrm{RT})}}{\sqrt{K_{k}^{(\mathrm{RT})}}},
$$

and under NLOS conditions by

$$
\eta_{\mathrm{NLOS}, k}^{(\mathrm{RT})}=\frac{\sigma_{k}^{(\mathrm{RT})}}{\sqrt{K_{k}^{(\mathrm{RT})}+1}} .
$$

Note that determining whether the mobile terminal is at an LOS or NLOS location is obvious in the RT simulations. We can thus obtain $K^{(\mathrm{RT})}, \theta_{k}^{(\mathrm{RT})}$, and $\eta_{k}^{(\mathrm{RT})}$ for all points in the $3 \mathrm{D}$ terrain and use for pinpointing the location of terminals, in combination with the results of the experimental data analysis.

\section{EXPERIMENTAL DATA ANALYSIS AND POSITION-DETERMINATION ACCURACY}

We now consider the application of the parameter estimation method described above to experimental data measured using array antennas. The accuracy of the proposed positiondetermination algorithm based on experimental data analysis is also discussed.

\subsection{Experimental conditions}

We analyzed data obtained from field testing in Yokosuka City, Japan, a city with a high housing density. An experimental array used as the base station receiver $(\mathrm{Rx})$ was mounted on top of a $15 \mathrm{~m}$ high building, employing the ULA with eight-element microstrip patch antenna. The antenna elements were separated by half the wavelength of the 


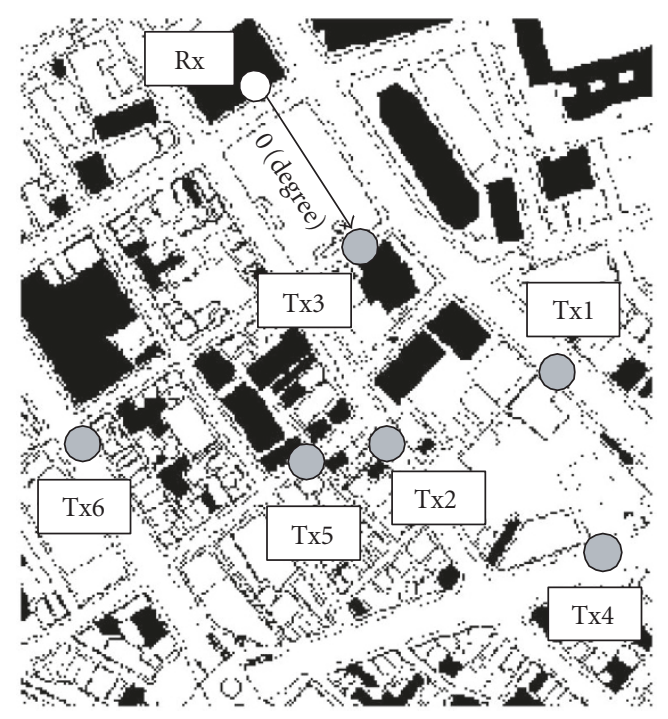

Figure 6: Map around testing area.

TABLE 1: Angle, distance, and transmitted power regarding each Tx.

\begin{tabular}{l|ccc|ccc}
\hline & \multicolumn{3}{|c|}{ LOS } & \multicolumn{3}{c}{ NLOS } \\
\cline { 2 - 7 } & Tx1 & Tx2 & Tx3 & Tx4 & Tx5 & Tx6 \\
\hline Angle (deg) & -15.7 & 10.6 & 0 & -6.5 & 22.9 & 54.8 \\
Distance (m) & 215 & 200 & 100 & 300 & 200 & 210 \\
Power $(\mathrm{dBm})$ & 0 & 10 & 0 & 30 & 20 & 30 \\
\hline
\end{tabular}

$2.335 \mathrm{GHz}$ carrier frequency. Figure 6 shows a map of the testing area, and Table 1 summarizes the angles, distances, and signal powers of the transmitters, which were $1.5 \mathrm{~m}$ high. The transmitters (Tx1-6) were stationary; three of them (Tx1 to $\mathrm{Tx} 3$ ) were at LOS positions, while the others ( $\mathrm{Tx} 4$ to $\mathrm{Tx} 6$ ) were at NLOS positions. The transmitted signal was formed by $\pi / 4$-shift QPSK modulation. We took 1900 snapshots at a sample rate of $2 \mathrm{MHz}$, which meant that the observation time was only $10^{-3}$ second. The other specifications and the experimental system are described elsewhere [34]. The data was collected at the base station. Note that the analysis was done for one terminal at a time.

\subsection{Experimental analysis}

The AOAs and DOSs were estimated by using the procedure described in Section 3.2.2. Tables 2 and 3 summarize the AOAs and DOSs estimated under LOS and NLOS conditions, respectively. We analyzed 1900 sample signals, divided into 19 groups, and calculated $E\left\{\left|\gamma_{k}\right|\right\}$ and $E\left\{\left|\phi_{k}\right|\right\}$ by averaging the estimates for those 19 periods to estimate the DOS, $\hat{\eta}_{k}$.

The previous numerical simulations [26] showed that the DOS was correlated with the BER of beamformed signals, which meant that the DOS indicated the degree of scattering. This is supported by the results shown in Tables 2 and 3 . The DOS of a direct path was much smaller than that of reflected ones since the definition of the DOS in (26) and (27) says that the DOS is smaller as the Ricean factor is larger. Thus, since both AOA and DOS are appropriate parameters for describing the characteristics of each scatterer, we use them as the key to obtain the locations of terminals.

\subsection{Positioning method and its accuracy}

We estimated the location of terminals using the results of the field testing and RT analysis by the method described in Section 2. First, using the RT simulator, pseudotransmitters were positioned at $10 \mathrm{~m}$ intervals within about $500 \mathrm{~m} \times 500 \mathrm{~m}$ on the map in Figure 6 and the AOAs and DOSs were estimated for each one. Note that the DOSs were obtained separately for the LOS and NLOS transmitter positions since the DOSs in the RT analysis behave differently in (29) and (30). The results were matched with the experimental analysis results by using the cost function of (1) with the weighting factor $v=0.5$.

Tables 4 and 5 show how accurately the location could be estimated in terms of probability for 200 trials using temporally different signals from the same point. For example, the location of Tx4 under NLOS conditions was estimated within $10 \mathrm{~m}$ in $31.5 \%$ of the trials, $20 \mathrm{~m}$ in $65.0 \%$, and $30 \mathrm{~m}$ in $83.5 \%$. Overall, the results show that positioning accuracy was within $30 \mathrm{~m}$ more than $73.5 \%$ of the time, even under NLOS conditions. These results easily satisfy the E-911 requirements of the FCC that the estimated location of a caller is within $50 \mathrm{~m}$ of the caller's actual location more than $67 \%$ of the time [2], and they show that our scheme outperforms other positioning schemes, such as $[13,17]$. 
TABLE 2: Parameter estimation results using actual data in LOS conditions.

\begin{tabular}{l|ccccc|ccc}
\hline Tx no. & \multicolumn{3}{|c|}{ Tx1 } & \multicolumn{3}{c|}{ Tx2 } & \multicolumn{2}{c}{ Tx3 } \\
\hline Path no. & Path1 & Path2 & Path1 & Path2 & Path3 & Path1 & Path2 & Path3 \\
\hline DOA (deg) & -15.7 & 45.7 & -24.6 & 10.3 & 17.5 & -38.8 & 0.0 & 40.5 \\
DOS & 0.0102 & 0.2942 & 0.0912 & 0.0535 & 0.5013 & 0.1492 & 0.0116 & 0.2239 \\
\hline
\end{tabular}

TABLE 3: Parameter estimation results using actual data in NLOS conditions.

\begin{tabular}{l|ccc|cc|ccc}
\hline Tx no. & \multicolumn{3}{|c|}{ Tx4 } & \multicolumn{3}{c|}{ Tx5 } & \multicolumn{2}{c}{ Tx6 } \\
\hline Path no. & Path1 & Path2 & Path3 & Path1 & Path2 & Path1 & Path2 & Path3 \\
\hline DOA (deg) & -18.2 & 12.5 & 44.8 & -29.0 & 15.1 & -40.1 & 3.1 & 49.4 \\
DOS & 0.0368 & 0.1674 & 0.0812 & 0.0952 & 0.0715 & 0.6824 & 0.1328 & 0.3972 \\
\hline
\end{tabular}

\subsection{Weighting factor and positioning accuracy}

To prove the effectiveness of introducing DOS, the positioning accuracy was evaluated at different values of the weighting factor $v$ in (1). Figure 6 shows the relationship between the probability of accuracy within $20 \mathrm{~m}$ and the weighting factor. The results confirm that introducing DOS, which reflects the propagation characteristics, dramatically improved position-determination accuracy. Although the optimization of the weighting factor is quite difficult since it depends on the transmitter location, the results show that the accuracy was approximately $15 \%$ to $40 \%$ better when both AOA and DOS were used than when only AOA was used.

\section{CONCLUSION}

We have described the novel method for determining the position of a wireless terminal; it uses a single array antenna and is suitable for use in multipath environments. It makes use of two spatial parameters, the angle of arrival and the degree of scattering, which reflect the path characteristics because they depend on the propagation environment between the transmitter and the receiver. These parameters are used in combination with the results of ray-tracing analysis with highly accurate 3D terrain data. The key features of our algorithm are that it is "blind," which needs no prior information about the transmitted signal such as signal waveform, keeping in mind the application of unknown source detection for radiowave surveillance. Furthermore, it is based on a local scattering model considering scattering in the vicinity of a mobile or some reflectors. We achieved a site-specific scheme with only a single base station by introducing the ray-tracing analysis.

Field testing showed that the proposed method was sufficiently accurate to meet the Federal Communications Commission requirements for mobile terminal position determination and that it outperformed other positioning algorithms, although the experimental area was only about $500 \mathrm{~m} \times 500 \mathrm{~m}$. This site-specific method can be used in other locations if only experimental data and 3D terrain data are available.

\section{APPENDIX}

The expectation of $\left|\gamma_{k}\right|$ in (19) is derived as follows. First we define $r=\left|\gamma_{k}\right|$, and the pdf $p(r)$ follows the Ricean distribution:

$$
p(r)=\frac{r}{\mu_{k}^{2}} \exp \left(-\frac{r^{2}+A_{k}^{2}}{2 \mu_{k}^{2}}\right) I_{0}\left(\frac{A_{k} r}{\mu_{k}^{2}}\right)
$$

where $\mu_{k}=L_{k} \cdot \operatorname{Var}\left\{\alpha_{\mathrm{Re}}\right\}=L_{k} \cdot \operatorname{Var}\left\{\alpha_{\mathrm{Im}}\right\}$, and $I_{0}(\cdot)$ is a zeroorder Bessel function of the first kind [33]. The expectation of $r$ is expressed as an integral in terms of $r$ :

$E\{r\}=\int_{0}^{\infty} r \cdot p(r) d r=\int_{0}^{\infty} \frac{r^{2}}{\mu_{k}^{2}} \exp \left(-\frac{r^{2}+A_{k}^{2}}{2 \mu_{k}^{2}}\right) I_{0}\left(\frac{A_{k} r}{\mu_{k}^{2}}\right) d r$.

This equation can be modified with the following mathematical formulae using a Gamma function and the Kummer's confluent hypergeometric function [33], respectively:

$$
\begin{gathered}
\int_{0}^{\infty} x^{\xi-1} \exp \left(-a^{2} x^{2}\right) I_{v}(b x) d x \\
=\frac{\Gamma\{(\xi+v) / 2\} b^{v}}{2^{v+1} a^{\xi+v} \Gamma(v+1)} \cdot M\left(\frac{\xi+v}{2} ; v+1 ; \frac{b^{2}}{4 a^{2}}\right), \\
M(c ; d ; z)=\sum_{k=0}^{\infty} \frac{(c)_{k}}{(d)_{k}} \frac{z^{k}}{k !}=1+\frac{c}{d} \frac{z}{1 !}+\frac{c(c+1)}{d(d+1)} \frac{z^{2}}{2 !} \\
+\frac{c(c+1)(c+2)}{d(d+1)(d+2)} \frac{z^{3}}{3 !}+\cdots,
\end{gathered}
$$

where $\Gamma(x)$ is the Gamma function, $M(c ; d ; z)$ is the Kummer's confluent hypergeometric function, and we define

$$
(x)_{n}=\frac{\Gamma(x+n)}{\Gamma(x)}=x(x+1) \cdots(x+n-1) .
$$


TABle 4: Positioning accuracy in LOS conditions: "Num." denotes the number of successful estimations within each accuracy up to 200 trials, and "Prob." is cumulative probability of correct positioning.

\begin{tabular}{l|rr|rr|rr}
\hline \multirow{2}{*}{ Positioning } & \multicolumn{2}{|c|}{ Tx1 } & \multicolumn{2}{c|}{ Tx2 } & \multicolumn{2}{c}{ Tx3 } \\
\cline { 2 - 7 } accuracy & Num. & Prob. & Num. & Prob. & Num. \\
\hline Within 10 m & 158 & $79.0 \%$ & 123 & $61.5 \%$ & 181 & $90.5 \%$ \\
Within 20 m & 40 & $89.0 \%$ & 49 & $86.0 \%$ & 19 & $100 \%$ \\
Within 30 m & 2 & $100 \%$ & 28 & $100 \%$ & 0 & $100 \%$ \\
\hline
\end{tabular}

TAble 5: Positioning accuracy in NLOS conditions: "Num." denotes the number of successful estimations within each accuracy up to 200 trials, and "Prob." is cumulative probability of correct positioning.

\begin{tabular}{|c|c|c|c|c|c|c|}
\hline \multirow{2}{*}{$\begin{array}{l}\text { Positioning } \\
\text { accuracy }\end{array}$} & \multicolumn{2}{|c|}{$\mathrm{Tx} 4$} & \multicolumn{2}{|c|}{ Tx5 } & \multicolumn{2}{|c|}{ Tx6 } \\
\hline & Num. & Prob. & Num. & Prob. & Num. & Prob. \\
\hline Within $10 \mathrm{~m}$ & 63 & $31.5 \%$ & 82 & $41.0 \%$ & 19 & $9.5 \%$ \\
\hline Within $20 \mathrm{~m}$ & 68 & $65.0 \%$ & 77 & $79.5 \%$ & 72 & $45.5 \%$ \\
\hline Within $30 \mathrm{~m}$ & 36 & $83.5 \%$ & 22 & $90.5 \%$ & 56 & $73.5 \%$ \\
\hline
\end{tabular}

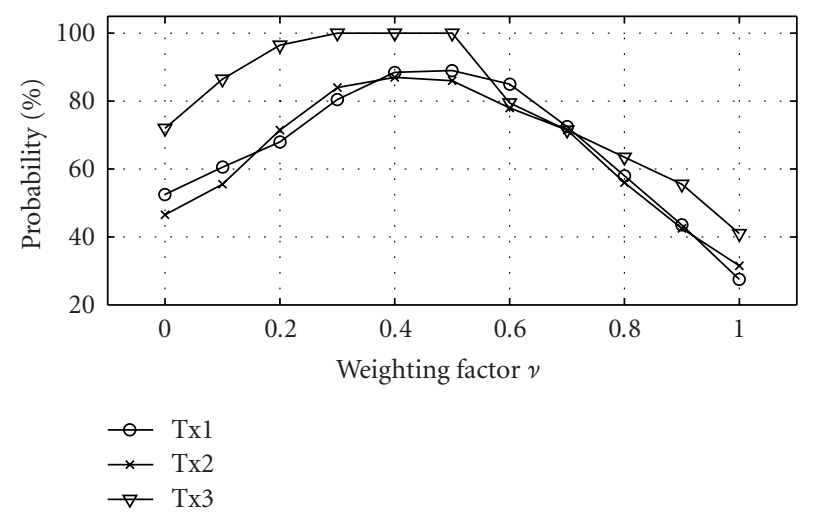

(a)

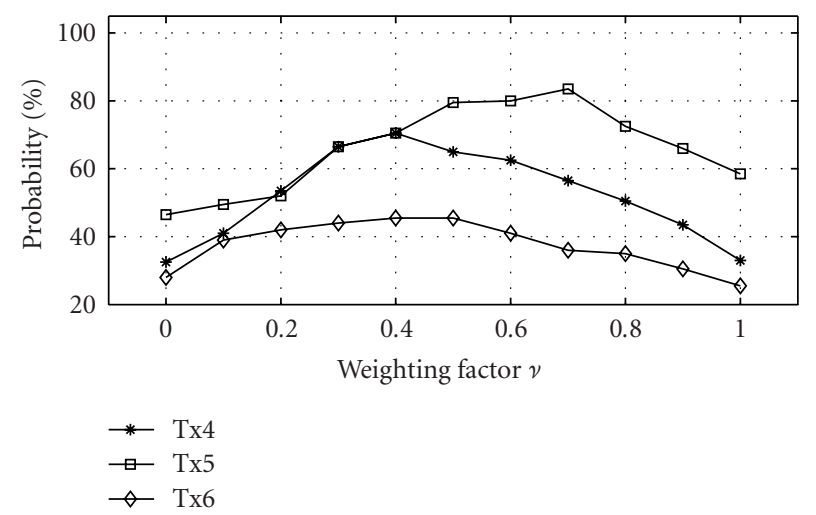

(b)

Figure 7: Positioning accuracy within $20 \mathrm{~m}$ in case of changing weighting factor $v$ : (a) the result of detecting Tx1 to 3 located at LOS positions, while (b) shows the detection probability of Tx4 to 6 at NLOS positions.
Substituting $x=r, \xi=3, v=0, a=1 /\left(\sqrt{2} \mu_{k}\right)$, and $b=A_{k} /$ $\mu_{k}^{2}$ into (A.3), we obtain (19) from (A.2) as

$$
E\left\{\left|\gamma_{k}\right|\right\}=\sqrt{\frac{\pi}{2}} \mu_{k} \exp \left(-\frac{A_{k}^{2}}{2 \mu_{k}^{2}}\right) M\left(\frac{3}{2} ; 1 ; \frac{A_{k}^{2}}{2 \mu_{k}^{2}}\right) .
$$

\section{REFERENCES}

[1] T. S. Rappaport, J. H. Reed, and B. D. Woerner, "Position location using wireless communications on highways of the futur," IEEE Communications Magazine, vol. 34, no. 10, pp. 33-41, 1996.

[2] J. H. Reed, K. J. Krizman, B. D. Woerner, and T. S. Rappaport, "An overview of the challenges and progress in meeting the E911 requirement for location service," IEEE Communications Magazine, vol. 36, no. 4, pp. 30-37, 1998.

[3] S. Tekinay, E. Chao, and R. Richton, "Performance benchmarking for wireless location systems," IEEE Communications Magazine, vol. 36, no. 4, pp. 72-76, 1998.

[4] http://www.tele.soumu.go.jp/e/monitoring/index.htm.

[5] C. Drane, M. Macnaughtan, and C. Scott, "Positioning GSM telephones," IEEE Communications Magazine, vol. 36, no. 4, pp. 46-54, 1998.

[6] S. U. Pillai, Array Signal Processing, Springer, New York, NY, USA, 1989.

[7] J. J. Caffery Jr. and G. L. Stüber, "Subscriber location in CDMA cellular networks," IEEE Transactions on Vehicular Technology, vol. 47, no. 2, pp. 406-416, 1998.

[8] Y. T. Chan and K. C. Ho, "A simple and efficient estimator for hyperbolic location," IEEE Transactions on Signal Processing, vol. 42, no. 8, pp. 1905-1915, 1994.

[9] I. Jami, M. Ali, and R. F. Ormondroyd, "Comparison of methods of locating and tracking cellular mobiles," in Proceedings of IEE Colloquium on Novel Methods of Location and Tracking of Cellular Mobiles and Their System Applications, vol. 99/046, pp. 1/1-6/1, London, UK, May 1999.

[10] A. J. Weiss, "On the accuracy of a cellular location system based on received signal strength measurement," IEEE 
Transactions on Vehicular Technology, vol. 52, no. 6, pp. 1508 1518, 2003.

[11] L. Cong and W. Zhuang, "Non-line-of-sight error mitigation in mobile location," IEEE Transactions on Wireless Communications, vol. 4, no. 2, pp. 560-573, 2005.

[12] R. J. Kozick and B. M. Sadler, "Source localization with distributed sensor arrays and partial spatial coherence," IEEE Transactions on Signal Processing, vol. 52, no. 3, pp. 601-616, 2004.

[13] S. Ahonen and P. Eskelinen, "Mobile terminal location for UMTS," IEEE Aerospace and Electronic Systems Magazine, vol. 18, no. 2, pp. 23-27, 2003.

[14] K. Kaemarungsi and P. Krishnamurthy, "Modeling of indoor positioning systems based on location fingerprinting," in Proceedings of the 23th Annual Joint Conference of the IEEE Computer and Communications Societies (INFOCOM '04), vol. 2, pp. 1012-1022, Hong Kong, March 2004.

[15] C. Nerguizian, C. Despins, and S. Affès, "Geolocation in mines with an impulse response fingerprinting technique and neural networks," in Proccedings of IEEE 60th Vehicular Technology Conference (VTC '04), vol. 5, pp. 3589-3594, Los Angeles, Calif, USA, September 2004.

[16] C. M. Takenga, K. R. Anne, K. Kyamakya, and J. C. Chedjou, "Comparison of gradient descent method, Kalman filtering and decoupled Kalman in training neural networks used for fingerprint-based positioning," in Proccedings of IEEE 60th Vehicular Technology Conference (VTC '04), vol. 6, pp. 41464150, Los Angeles, Calif, USA, September 2004.

[17] M. Porretta, P. Nepa, F. Giannetti, et al., "A novel single base station location technique for microcellular wireless networks: description and validation by a deterministic propagation model," IEEE Transactions on Vehicular Technology, vol. 53, no. 5, pp. 1502-1514, 2004.

[18] M. C. Vanderveen, A.-J. van der Veen, and A. Paulraj, "Estimation of multipath parameters in wireless communications," IEEE Transactions on Signal Processing, vol. 46, no. 3, pp. 682690, 1998.

[19] D. N. Knisely, S. Kumar, S. Laha, and S. Nanda, "Evolution of wireless data services: IS-95 to cdma2000," IEEE Communications Magazine, vol. 36, no. 10, pp. 140-149, 1998.

[20] D. Asztély and B. Ottersten, "The effects of local scattering on direction of arrival estimation with MUSIC," IEEE Transactions on Signal Processing, vol. 47, no. 12, pp. 3220-3234, 1999.

[21] T. Trump and B. Ottersten, "Estimation of nominal direction of arrival and angular spread using an array of sensors," Signal Processing, vol. 50, no. 1-2, pp. 57-69, 1996.

[22] M. Wax and O. Hilsenrath, "Signature matching for location determination in wireless communication systems," US patent no. $6,112,095$.

[23] M. C. Lawton and J. P. McGeehan, "The application of a deterministic ray launching algorithm for the prediction of radio channel characteristics in small-cell environments," IEEE Transactions on Vehicular Technology, vol. 43, no. 4, pp. 955969, 1994.

[24] M. P. M. Hall, L. W. Barclay, and M. T. Hewitt, Propagation of Radiowaves, The Institution of Electrical Engineers, London, UK, 1996.

[25] H. Tsuji, K. Yamada, and H. Ogawa, "A new approach to array beamforming using local scattering modeling," in Proccedings of IEEE 54th Vehicular Technology Conference (VTC'01), vol. 3, pp. 1284-1288, Atlantic City, NJ, USA, October 2001.

[26] K. Yamada and H. Tsuji, "Using a model of scattering in a lowintersymbol-interference channel for array beamforming," in
Proceedings of the 11th European Signal Processing Conference (EUSIPCO '02), Toulouse, France, September 2002.

[27] H. Yan and H. H. Fan, "On source association of DOA estimation under multipath propagation," IEEE Signal Processing Letters, vol. 12, no. 10, pp. 717-720, 2005.

[28] S. Kikuchi, H. Tsuji, R. Miura, and A. Sano, "Mobile localization using local scattering model in multipath environments," in Proccedings of IEEE 60th Vehicular Technology Conference (VTC '04), vol. 1, pp. 339-343, Los Angeles, Calif, USA, September 2004.

[29] M. Wax and T. Kailath, "Detection of signals by information theoretic criteria," IEEE Transactions on Acoustics, Speech, and Signal Processing, vol. 33, no. 2, pp. 387-392, 1985.

[30] Y. Abramovich, N. K. Spencer, and A. Y. Gorokhov, "Bounds on maximum likelihood ratio-part II: application to antenna array detection - estimation with imperfect wavefront coherence," IEEE Transactions on Signal Processing, vol. 53, no. 6, pp. 2046-2058, 2005.

[31] S. Shahbazpanahi, S. Valaee, and M. H. Bastani, "Distributed source localization using ESPRIT algorithm," IEEE Transactions on Signal Processing, vol. 49, no. 10, pp. 2169-2178, 2001.

[32] T.-J. Shan, M. Wax, and T. Kailath, "On spatial smoothing for direction-of-arrival estimation of coherent signals," IEEE Transactions on Acoustics, Speech, and Signal Processing, vol. 33, no. 4, pp. 806-811, 1985.

[33] Z. X. Wang and D. R. Guo, Special Functions, World Scientific, Hackensack, NJ, USA, 1989.

[34] A. Kanazawa, H. Tsuji, H. Ogawa, Y. Nakagawa, and T. Fukagawa, "An experimental study of DOA estimation in multipath environment using an adaptive array antenna equipment," in Proceedings of the Asia-Pacific Microwave Conference, pp. 804807, Sydney, Australia, December 2000.

Shohei Kikuchi received the B.E., M.E., and Ph.D. degrees in System Design Engineering from Keio University, Japan, in 2002, 2003, and 2006, respectively. Since April 2006, he has been working for Toshiba Corporation. His research interests are in array signal processing and its applications for mobile communication systems. He received a Young Researcher's Encouragement Award from IEEE VTS Japan in 2003. He is a Member of IEEE, IEICE, and SICE.

Akira Sano received the B.E., M.E., and Ph.D. degrees in mathematical engineering and information physics from The University of Tokyo, in 1966, 1968, and 1971, respectively. He is currently a Professor Department of System Design Engineering, Keio University. He was a Visiting Research Fellow at the University of Salford, Salford, UK, from 1977 to 1978 . His research interests include adaptive modeling and design

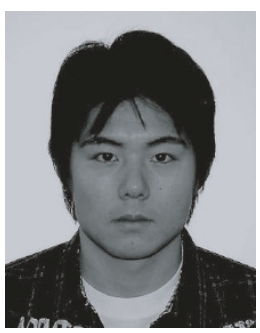
theory in control, signal processing, and communications, and applications to control of sounds and vibrations, mechanical systems, and mobile communication systems. He received the Kelvin Premium from the Institute of Electrical Engineering in 1986. He is a Fellow of the Society of Instrument and Control Engineers and is a Member of the Institute of Electrical Engineering of Japan and the Institute of Electronics, Information, and Communications Engineers of Japan. He was General Cochair of 1999 IEEE Conference of 
Control Applications and served as Chair of IFAC Technical Committee on Modeling and Control of Environmental Systems from 1996 to 2001. He has also been Vice Chair of IFAC Technical Committee on Adaptive Control and Learning since 1999 and has been Chair of the IFAC Technical Committee on Adaptive and Learning Systems since 2002.

Hiroyuki Tsuji received the B.E., M.E., and Ph.D. degrees from Keio University in 1987, 1989, and 1992, respectively. Since 1992, he has been working in the National Institute of Information and Communications Technology (NICT), Independent Administrative Institution, Japan. In 1999, he was a Visiting Researcher at University of Minnesota. His research interests are in array signal processing, particularly as applied to commu-

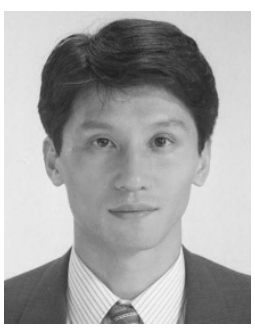
nications. He received the IEICE 1996 Young Engineer Award. He is a Member of IEEE and IEICE. 